\title{
Water flow regulator for irrigation canals
}

\author{
Dmitry Benin, and Vera Snezhko* \\ Russian State Agrarian University - Moscow Timiryazev Agricultural Academy, Moscow, Russia
}

\begin{abstract}
A distinctive feature of the irrigated systems of the Russian Federation is a large area of irrigated areas and a significant length of irrigation canals. With a large area of the irrigated massif on small canals, the use of electricity to regulate the throughput of network hydraulic structures is economically ineffective. Preference is given to means of hydraulic automation of water supply. Regulation of the throughput of a hydraulic structure is based on the laws of fluid flow within it. On irrigation canals in Russia, as in world practice, water-operated gates are widely used. One of the disadvantages of such gates is moving metal parts and sensors, which reduce the operational reliability of structures. A new regulator of throughput is proposed, the action of which is based on the injection effect. Compression of the flow by physical elements was replaced by the circulation of surplus water supply between the outlet section of the water supply structure and the downstream. The regulator is built into the pressure drop between the high and low order channels. Regulating the throughput of the tubular water outlet automatically begins after shutting down one or more sprinklers that take water from the lower order canal. After turning the sprinklers into operation, the regulator automatically restores the original throughput. Using the theory of jet pumps, a new method has been developed for the theoretical determination of the main hydraulic characteristics of the regulator. These include the size of the nozzle and the velocities of the injection and injection streams. The derivation of theoretical dependencies was based on the classical equations of fluid mechanics; the flow within the structure was considered quasi-one-dimensional. The obtained calculated dependencies were verified using numerical and physical modeling. The data of the physical and numerical experiment were in good agreement with the theoretical dependences. Further optimization of the controller can be performed by changing its geometric parameters.
\end{abstract}

\section{Introduction}

In the Russian Federation, irrigated lands occupy more than 4.6 million hectares, which is $41 \%$ of the total area of ameliorated lands. According to the Federal State Statistics Service, 7.5 billion cubic meters of fresh water was used in 2019 for irrigation and agricultural water supply. Reclamation waterworks, created in the middle of the last century, currently need reconstruction [1]. On old irrigation systems, water supply automation tools are rarely used.

*Corresponding author:VL_Snejko@mail.ru 
This leads to large volumes of excess water discharge and an increase in the value of products grown on irrigated land.

Many countries face the problem of rational distribution and saving water in irrigation systems. In South Korea, during the operation of a cascade of irrigation reservoirs, the volume of return flow from irrigated rice fields can be up to a third of the water supply. [2]. The large volume of water discharged from the canals of the rice systems and the lack of management of water distribution are factors that reduce the efficiency of irrigation for farms in Brazil. When supplying water from the main to the secondary canal, sluice gates are traditionally used here. The controlled change in the flow rate of these valves is $20 \%$. Fiberglass water regulators with the movable seal have an adjustable flow rate change of only $5 \%$ [3].

Automatic movable gates are installed in the inlet heads of culverts and drainage pipes [4]. In irrigation, hydro-automatic gates are used, which include the Begeman gate [5] (or flap gate) and the Romaine gate, operating under free flow conditions [6]. For the transition to a new steady state when regulating, the gates need some time. The weather vane shutter can operate with flooded outflow [7].

Swing gates can carry out automatic control of the water level in the upper channel. The basic configurations of these gates were developed in the Netherlands at the beginning of the 20th century and are used in California, Indonesia, Nigeria, and the Dominican Republic [8]. A gate for regulating the water level of hydromechanical action is presented in [9]. The study of the possibilities of automating cotton irrigation in furrows using water supply with siphons is given in [10].

Analysis shows that regulators using automatic movable gates are most common on irrigation canals. Malfunctions in the operation of sensors and actuators in automated irrigation canals are one of the problems of their operation [11].

In Russia, on large irrigation (main) canals with flow rates over $30 \mathrm{~m}^{3} / \mathrm{s}$, complex means of automation of electrical action are used. This corresponds to the established world practice. Sophisticated control systems, including electronics, are used to distribute water in the basin of the main irrigation canal [12]. With a large area of the irrigated massif on small canals with flow rates from 1 to $10 \mathrm{~m}^{3} / \mathrm{s}$, the use of electricity is economically ineffective. Here it is advisable to use the means of hydraulic automation of water supply. The control principle of the automatic hydraulic action depends on the control scheme for water distribution between consumers. This process is based on changing a specific parameter: water flow rate or water level in the canal.

On the irrigation canals, there are always hydraulic structures designed to pass water through various obstacles. These include siphons, pipe crossings, drops. It is proposed to use the features of the water flow in these structures to purposefully change their throughput. Unlike gate-type regulators, the principle of water flow control is based on the hydrodynamic characteristics of the flow inside the structures. Regulators of the new type do not have to move mechanical parts and level sensors. They turn on automatically when water consumption in the tailwater decreases and turn off when it further increases. The presence of operating personnel is not required to start the regulation process. The diagram of the new regulator, combined with the pressure drop on the channels, is shown in Figure 1. 


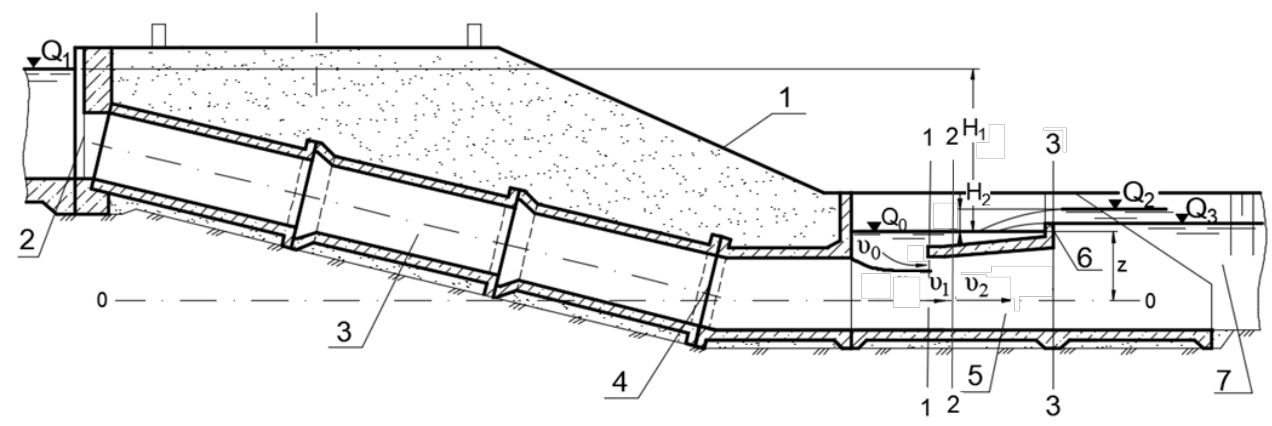

Fig. 1. Regulator circuit: 1 is dam; 2 is shutter; 3 is culvert; 4 is horizontal section; 5 is diffuser; 6 is weir threshold; 7 is outlet channel.

The pressure drop from the pipes 3 is laid under the dam 1 and connects the higher-order canal with the lower order irrigation canal 7. Water from the irrigation canal is taken by sprinklers. Shutter 2 serves only for a complete overlap of the structure. The horizontal pipe section 4 is followed by a diffuser 5 . At the maximum water consumption in channel 7 , the water level ${ }_{\nabla} Q_{3}$. is set. The spillway threshold 6 is located at this mark.

After shutting down one or more sprinklers, the water level in channel 7 will rise to the mark ${ }_{\nabla} Q_{2}$. The regulation process starts automatically. Excess water supply through the weir 6 is fed to the diffuser cover. An intermediate pool with a mark ${ }_{\nabla} Q_{0}$. is formed. The flow velocity $v_{1}$ out of the pipe is much higher than the flow velocity $v_{0}$. Water from the intermediate pool is taken in through an opening in the diffuser cover. Mixing of streams occurs. The flow with velocity $v_{2}$ enters the diffuser and then into the channel. The throughput of the pressure drop pipe decreases due to additional energy losses. In the process of regulation, excess water circulates between the tailwater and the outlet section of the pipe 4. When the sprinklers are switched on, the water level in the channel 7 again drops to the mark ${ }_{\nabla} \mathrm{Q}_{3}$. Water stops flowing through the weir to the diffuser cover. The regulation process is terminated. The details of the parameters of diffuser 5 are shown in Figure 2.

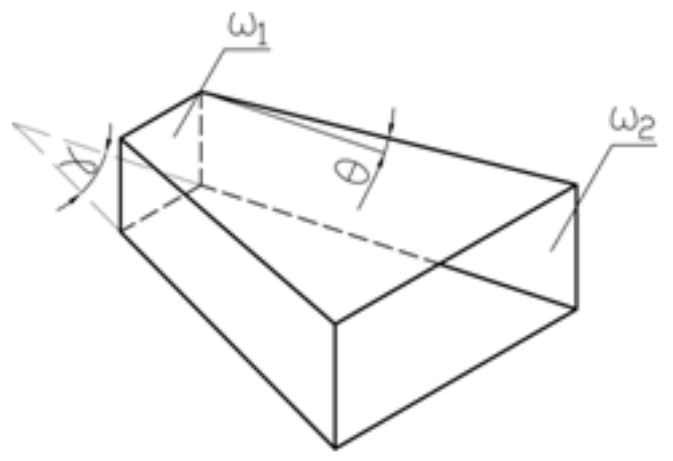

Fig. 2. Diffuser design

For further research, the following geometric characteristics of the diffuser were taken: expansion ratio $n_{l}=2$, horizontal expansion angle $\gamma=6^{\circ}$, vertical expansion angle $\theta 5^{\circ}$. The coefficient of hydraulic resistance of the diffuser $\zeta=0,32$ was determined experimentally, taking into account the energy losses when the flow enters the downstream volume. The flow within the diffuser was continuous. The expansion angle of the diffuser is close to the optimum value. 
The aim of the research was the development of a new method for the theoretical determination of the hydraulic characteristics of the proposed regulator and the verification of the obtained calculated dependencies according to the data of a physical (hydraulic) experiment.

\section{Methods}

For the first time, it is proposed to consider the hydraulic circuit of the regulator operation as an injection process. The injection is the continuous mixing of two streams of liquid, in which the injecting (working) stream transfers part of its energy to the injected stream. In the regulator, the injector is the flow coming from the upstream to the downstream. The injected flow is the flow coming from the downstream side to the diffuser cover. In this case, the speed of the working flow $v_{1}$ must exceed the speed of the injected flow $v_{2}$ at least two times (Figure 1). In the process of injection, the passive flow is raised by the working flow from the ${ }_{\nabla} Q_{0}$ mark to the ${ }_{\nabla} Q_{2}$. mark. The regulator is somewhat similar to an asymmetric water jet pump located under the dam.

An integral part of injection devices is the presence of a compressed section, behind which the working flow draws the injected medium into the mixing chamber. In this case, there is no nozzle. Its role is played by the interface between flows in the section 1-1. The mixing chamber is a horizontal insert between sections 1-1 and 2-2. The diffuser equalizes the total flow velocity after the chamber and before entering the channel. It can also increase the throughput of the injector. The main characteristics of injection are the nozzle size and the speed of the injecting and injected flows. These parameters are determined in the course of research.

The injection effect has been known since the 16th century, but it became widely used in technology only at the beginning of the 20th century. Currently, mixing flows in jet pumps is used for the separation of media in the mining industry [13], oil production [14], thermoacoustic engines and refrigerators [15].

The earliest works on the theory of jet pumps and the study of their parameters belong to F. Watson [16], J. Gosline and M. O'Brien [17], R. Vogel [18], B.E. Friedman [19]. The development of the theory of jet pumps considering the compressibility of a liquid is given by T. W. Van Der Lingen [20]. R. G. Cunningham [21] describes a one-dimensional model of mixing flows and exit into a diffuser in the presence of friction. He also studies the influence of the shape of the nozzle, the length of the mixing chamber and the distance between them on the parameters of the gas-jet pump [22]. The basic equations for the performance of fluid transfer pumps and their optimization are set out by $\mathrm{Hu}$ Xianghan [23].

The derivation of theoretical dependencies given below is based on equations of fluid mechanics: balances of flow and energy, momentum, Bernoulli's equations. The premises of the quasi-one-dimensional flow theory are applied.

\section{Results and Discussion}

The flow rate $Q_{2}$ enters the section 1 - 1 at the entrance to the mixing chamber with area $F_{2}$. It is equal to the sum of the flow rate $Q_{1}$, and the flow rate $Q_{0}$ injected from the intermediate pool. It is assumed that the flows in this section are not yet mixed. Then the area occupied by the flow rate $\mathrm{Q}_{0}$ is equal to:

$$
F_{0}=F_{2}-F_{1}
$$


The difference between the water levels in the headwater and the control tank is equal to $\mathrm{H}_{1}$. The difference between the water levels downstream and the control tank is $\mathrm{H}_{2}$. The distance between the axis of the horizontal section of the pipe and the water level in the control tank is equal to $Z$. This value is analogous to the immersion of the axis of the jet pump. A diffuser begins behind the mixing chamber in section $2-2$, the resistance coefficient of which is $\zeta$.

In the horizontal section of the regulator, a pressure pulse acts on the fluid along the flow axis. In the mixing chamber between sections $1-1$ and $2-2$, the second pulse of pressure forces is equal to the increment in the momentum of the fluid:

$$
F_{2} \cdot\left(P_{2}-P_{1}\right)=\rho_{1} \cdot Q_{1} \cdot \vartheta_{1}+\rho \cdot Q_{0} \cdot \vartheta_{0}-\rho \cdot Q_{2} \cdot \vartheta_{2}
$$

where $\mathrm{F}_{2}$ is the area of the mixing chamber, $\mathrm{Q}_{2}$ is the total flow rate, $v_{2}$ is the flow rate at the end of the mixing chamber, $\rho$ is the density of water.

If each of the streams occupies the area $\mathrm{F}$, then the expressions are valid:

$$
\begin{gathered}
F_{2}=\frac{Q_{2}}{\vartheta_{2}} \\
Q_{1}=F_{1} \cdot \vartheta_{1} \\
Q_{0}=F_{0} \cdot \vartheta_{0}
\end{gathered}
$$

Substitution of the above expressions into the momentum equation and simple mathematical transformations lead to the first equation of the system:

$$
\frac{P_{2}-P_{1}}{\rho}=\vartheta_{2} \cdot\left(\frac{F_{1} \cdot \vartheta_{1}^{2}+F_{0} \cdot \vartheta_{0}^{2}}{F_{1} \cdot \vartheta_{1}+F_{0} \cdot \vartheta_{0}}-\vartheta_{2}\right)
$$

It is assumed that at the entrance to the mixing chamber, the flows $\mathrm{Q}_{1}$ and $\mathrm{Q}_{2}$ are practically parallel and have a small curvature. Mixing chamber entry losses are not included. Therefore, the pressure $\mathrm{P}_{1}$ at the inlet to the mixing chamber is the same for both flows $\mathrm{Q}_{1}$ and $\mathrm{Q}_{2}$. The second and third equations of the system are obtained:

$$
\begin{aligned}
& Z=\frac{P_{1}}{\rho g}+\frac{\vartheta_{0}^{2}}{2 g} \\
& H_{1}+Z=\frac{P_{1}}{\rho g}+\frac{\vartheta_{1}^{2}}{2 g}
\end{aligned}
$$

Bernoulli's equation for the pressure at the outlet from the mixing chamber to the diffuser is the fourth equation in the system:

$$
\frac{P_{2}}{\rho g}=Z+H_{2}-(1-\xi) \cdot \frac{\vartheta_{2}^{2}}{2 g}
$$

The continuity equation for the velocity at the outlet from the mixing chamber is the fifth equation of the system:

$$
\vartheta_{2}=\frac{Q_{1}+Q_{0}}{F_{2}}=\frac{F_{1} \cdot \vartheta_{1}+F_{0} \cdot \vartheta_{0}}{F_{2}}
$$

To solve the resulting system of equations (1-5), pressures $\mathrm{p}_{1}$ and $\mathrm{p}_{2}$ are excluded from equation (1). Then the speeds $v_{0}$ and $v_{2}$ are expressed in terms of the speed $v_{1}$. The further joint solution of the equations of the system makes it possible to obtain the basic calculated dependences for a controller with an injection effect. 
Injection flow rate:

$$
v_{1}=\left(\frac{2 \cdot g \cdot H_{1} \cdot \mathrm{B}^{2}-2 \cdot \mathrm{A} \cdot \mathrm{C}+\sqrt{\left(2 \cdot \mathrm{A} \cdot \mathrm{C}-2 \cdot g \cdot H_{1} \cdot \mathrm{B}^{2}\right)^{2}+4 \cdot \mathrm{C}^{2} \cdot\left(\mathrm{B}^{2}-\mathrm{A}^{2}\right)}}{2 \cdot\left(B^{2}-A^{2}\right)}\right)^{1 / 2}
$$

Injection flow rate:

$$
v_{2}=\frac{F_{1} \cdot \vartheta_{1}+F_{0} \cdot\left(\vartheta_{1}^{2}-2 g H_{1}\right)^{1 / 2}}{F_{2}}
$$

where $\mathrm{A}$ and $\mathrm{B}$ are constants depending on the design features of the flow path of the regulator:

$$
\begin{aligned}
& A=\frac{F_{1}}{F_{2}}+\frac{F_{0}}{F_{2}}-\left(\frac{F_{1}}{F_{2}}\right)^{2}-\left(\frac{F_{0}}{F_{2}}\right)^{2}-\frac{1}{2}-\frac{(\xi-1)}{2} \cdot\left[\left(\frac{F_{1}}{F_{2}}\right)^{2}+\left(\frac{F_{0}}{F_{2}}\right)^{2}\right] \\
& B=(1-\xi) \cdot \frac{F_{1}}{F_{2}} \cdot \frac{F_{0}}{F_{2}}-2 \cdot \frac{F_{1}}{F_{2}} \cdot \frac{F_{0}}{F_{2}}
\end{aligned}
$$

$\mathrm{C}$ a constant additionally related to the position of the bays:

$$
C=g H_{2}+g H_{1} \cdot\left[2 \cdot \frac{F_{0}}{F_{2}}-1-\left(\frac{F_{0}}{F_{2}}\right)^{2} \cdot(\xi+1)\right]
$$

Expressions (6-8) contain the areas occupied by the flows $F_{1}$ and $F_{0}$. Their values are determined for a specific design of the regulator by analyzing the interface between the flows $Q_{1}$ and $Q_{0}$ in section 1-1. Therefore, it was necessary to conduct a hybrid experiment, which included a physical experiment and further numerical simulation of the flow.

In the course of a physical experiment in a hydraulic chute, the operation of a model of a plexiglass regulator, made on a scale of 1:10, was studied. The tray is divided into pits. The injection flow entered the head of the structure. The injected flow entered from the downstream through the weir on the diffuser cover. The hole area in the upper wall of the pipe is equal to the diameter of the pipe. The mixing chamber length is equal to two pipe diameters. The diffuser is pyramidal with a degree of expansion $\mathrm{n}_{1}=2$ (Figure 2).

The similarity of pressure flows corresponds to the equality of the coefficient of hydraulic friction $\lambda$ (Darcy coefficient) of the model and nature. The hydraulic equivalent roughness of the model walls is $\Delta=0.03 \mathrm{~mm}$. This is the hydraulically equivalent roughness of concrete $\Delta=0.3 \mathrm{~mm}$, reduced by a factor of 10 . The model provided a turbulent flow regime with Reynolds numbers $R e=1.1 \cdot 10^{5} \div 1.6 \cdot 10^{5}$. This corresponds to a self-similar region in terms of the Reynolds number, within which the viscosity does not affect the local resistance coefficients. The movement of fluid in the regulator from the upstream to the downstream is influenced by gravity. In this case, the Froude number was the only and defining criterion for modeling turbulent characteristics.

The accuracy of measuring the flow parameters in the experiment was estimated by the limiting relative total error. The boundaries of the confidence interval for it are calculated with a confidence level of $95 \%$. For the flow depth, the limiting error was in the range $(0.6$ - 2.9$) \%$, for the flow rate $(1.02-2.5) \%$, for the piezometric pressure $(0.2-1.9) \%$.

The hydraulic characteristics of the regulator measured on the physical model were substituted into the numerical model as boundary conditions. The STAR-CCM + CFD package was used to model the flow between the pits. The $k-\varepsilon$ turbulence model is used. These models have proven themselves well in the numerical simulation of jet devices [24]. 
The CFD model was verified by comparing the experimental data and the data obtained from calculations. The computational mesh was generated using a prismatic layer in the near-wall flow region. In the mixing zone of the flows, the mesh was thickened. The free surface was modeled as a two-phase interaction with gravity. The cell size in the model was taken to be $2.5 \mathrm{~mm}$. This value corresponded to the greatest coincidence in the control sections of the velocities and pressures of the physical and numerical models.

The position of the boundary streamline was associated with the ratio $\frac{Q_{1}}{Q_{0}}$. In the experiments, the $\frac{F_{1}}{F_{2}} \quad$ value was within $0.75 . .0 .8$, while the $\frac{Q_{1}}{Q_{0}}$ ratio ranged from 5 to 13 . The injection flow rate of the regulator $\mathrm{v}_{-} 1$ in all experiments exceeded the injected flow rate v_0 by $1.5 \ldots 2.8$ times.

To verify the calculated theoretical dependencies (6) and (7), the data obtained on the physical model of the controller were compared with the theoretical curve (Figure 3). Due to a slight change in the area of the injection flow in the mixing chamber, the calculations were performed for a constant value $\frac{F_{1}}{F_{2}}=0.78$. The calculations are performed, taking into account the fact that $Q_{1}=F_{1} \cdot \vartheta_{1}$ and $Q_{0}=F_{0} \cdot \vartheta_{0}$.

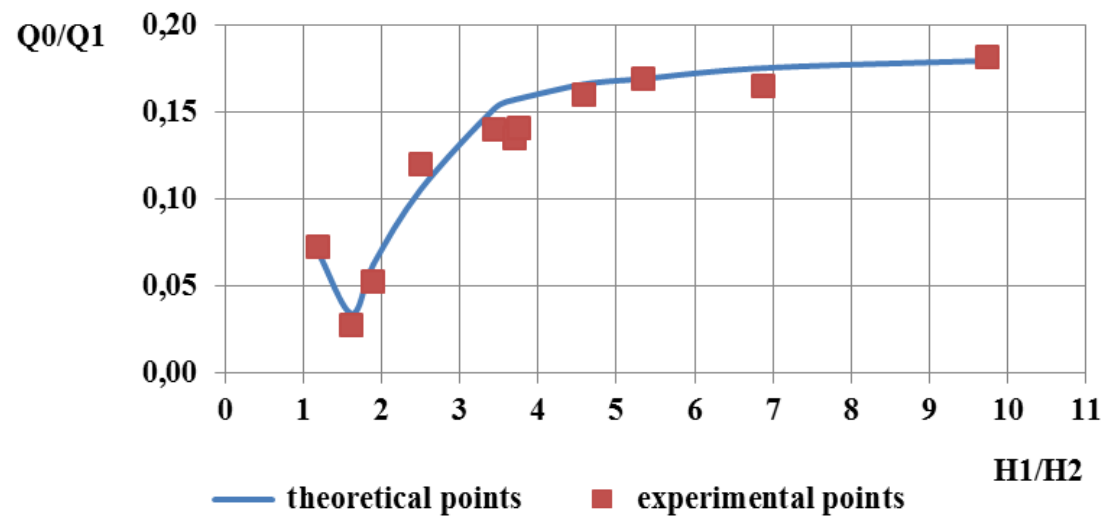

Fig. 3. Verification of theoretical dependencies

The data of theoretical calculations and physical experiment showed sufficient agreement. The right border of the graph (Figure 3) corresponds to high values of the depth difference between the wells. At the same time, the water level the upstream is significantly higher than the water level the downstream.

The pressure on the structure is equal to $\mathrm{H}=\mathrm{H}_{1}-\mathrm{H}_{2}$. Consequently, the ratio of heads above the level of the intermediate water is always greater than one $\frac{\mathrm{H}_{1}}{\mathrm{H}_{2}}>1$. This is due to the presence of energy losses during the motion of the injection flow. At large depth differences between the tailwater and the outlet section of the pipe, up to $20 \%$ of the excess water supply can circulate. At small depth differences, this value is about $15 \%$.

The characteristics of the regulator as an injection device are closely related to the length of the mixing chamber and the characteristics of the diffuser. Varying the specified geometric parameters will further optimize the regulatory capacity of the structure.

The water flow to the diffuser cover depends on the head above the weir threshold. This head is determined by an increase in the depth in the channel with a decrease in water consumption. Ensuring the accuracy of regulation is based on matching the length of the spillway front and the water level in the intermediate pool, to which all heads are tied. The 
volume of water in the intermediate pool must provide the required water depth in the space above the diffuser to maintain a given injection flow rate $v_{0}=f\left(H_{1}, H_{2}\right)$.

\section{Conclusions}

1. A flow regulator for irrigation canals is developed. The structure is located under the dam at the drop of the main and secondary canals.

2. The regulator can be obtained from a pressure drop pipe after equipping the outlet section of the pipe with a diffuser with a small expansion angle.

3. Using the theory of injection, a new method for determining the hydraulic parameters of the regulator has been developed.

4. To verify the theoretical equations, a physical and numerical experiment was performed.

5. The possibility of using the injection effect when regulating the throughput of an irrigation canal has been proved.

\section{Acknowledgments}

This work was supported by the Ministry of Science and Education of the Russian Federation under agreement No. 075-15-2020-905 dated November 16, 2020 on the provision of a grant in the form of subsidies from the federal budget for the implementation of state support for the creation and development of a world-class scientific center "Agricultural technologies of the future".

\section{References}

1 Kozlov D.V., Simonovich O.S., and Snezhko V.L. 2020 The Failure Rate of LowHead Ground Dams of Dangerous and Unsatisfactory Safety Level Power Technology and Engineering 54, pp 343-348, (2020), DOI: 10.1007/s10749-02001213.

2 Kang M.G., Park S.W. Modeling water flows in a serial irrigation reservoir system considering irrigation return flows and reservoir operations Agricultural WaterManagement, Elsevier vol. 143(C) pp. 131-141, (2014)

3 Do Amaral L G H Righes A A E Sousa Filho P D S \& Costa, R D 2005 Automatic regulator for channel flow control on flooded rice Agricultural Water Management Vol 75 Issue 3 pp 184-193, (2004)

4 Kubrak J Kubrak E Kaca E Kiczko A and Kubrak M. Theoretical and Experimental Analysis of Operating Conditions of a Circular Flap Gate for an Automatic Upstream Water Level Control Water 11 pp. 2576, (2019), DOI: 10.3390/w11122576.

5 Litrico X., Belaud G., Baume J and Ribot-Bruno J, Hydraulic Modeling of an Automatic Upstream Water-Level Control Gate Journal of Irrigation and Drainage Engineering-asce ,131, pp. 176-189, (2005)

6 Hou Caishui. Numerical simulation of hydraulic characteristics of hydro-automatic Gate, Proceedings International Conference on Mechatronic Sciences, Electric Engineering and Computer (MEC) Shenyang China pp 2739-42, (2013) DOI: 10.1109/MEC.2013.6885493.

7 Belaud G Litrico X de Graaff B \& Baume J Hydraulic Modeling of an Automatic Upstream Water Level Control Gate for Submerged Flow Conditions 2008 Journal of Irrigation and Drainage Engineering-asce 134 pp. 315-326 DOI: 10.1061 / (ASCE) 0733-9437 (2008) 134: 3 (315). 
8 Burt C Angold R Lehmkuhl M \& Styles S 2001 Flap gate design for automatic upstream canalwater level control Journal of Irrigation and Drainage Engineeringasce 127 pp 84-91, (2001), DOI:10.1061/(ASCE)0733-9437(2001)127:2(84).

9 Cassan L Baume J Belaud G Litrico X Malaterre P \& Ribot-Bruno J 2011 Hydraulic Modeling of a Mixed Water Level Control Hydromechanical Gate Journal of Irrigation and Drainage, Engineering-asce 137, pp, 446-453

10 Koech R K Smith R J \& Gillies M H 2010 Furrow irrigation in the Australian cotton industry: alternative water delivery systems and their potential for automation National Centre for Engineering in Agriculture Report No 1002982/1 University of Southern Queensland, Toowoomba Australia

11 Choy S Weyer E Reconfiguration schemes to mitigate faults in automated irrigationchannels: Experimental results Proceedings of the 45th IEEE Conference on DecisionandControl San Diego CA USA pp 2189-94, (2006),

12 Feliu V Rivas-Perez R Castillo G \& Fernando J. Fractional order controller robust to timedelay for water distribution in an irrigation main canal pool Computers and Electronics in Agriculture 69 pp 185-197, (2009)

13 Dueck J G Pikushchak E V \& Minkov L L. Modelling of change of the classifier separation characteristics by water injection into the apparatus. Thermophys.Aeromech. 16, pp.247-258, (2009)

14 Zhao J Dong Y Fu J Zhao L \& ZhangY 2020 Design and experiment of energy-saving water injection pump Journal of Petroleum Exploration and Production Technology10(5), pp. 2127-35, (2020) DOI: 10.1007/s13202-020-00860-1.

15 Geng Q Zhou G \& Li Q. The influence of reynolds numbers on resistance properties of et pumps In AIP Conference Proceedings Vol 1573, pp. 514-519, American Institute of PhysicsInc, (2014)

16 Watson F R B. The Production of a Vacuum in an Air Tank by Means of a Steam Jet Proceedings of the Institution of Mechanical Engineers 124(1) pp 231-300, (1993) DOI:10.1243/pime_proc_1933_124_012_02.

17 Gosline J O'Brien M The Water Jet Pump. University of California Publications in Engineering Vol 3 No 3 pp 167-190, (1934)

18 Vogel R. Theoretical and experimental investigation of air ejectors Maschinenbautechnik Berlin 5 pp 619-637, (1956)

19 Fridman B E. Gidroelevatory [Hydroelevators] Moscow Mashgiz Publ (1960)

20 Van Der Lingen T W. A jet pump design theory. Journal of Fluids Engineering, Transactions of the ASME 82(4) pp 947-960, (1960) DOI: $10.1115 / 1.3662812$.

21 Cunningham R G. Gas compression with the liquid jet pump Journal of FluidsEngineering Transactions of the ASME 96(3) pp 203-215, (1974), DOI: 10.1115/1.3447143.

22 Cunningham R G. Dopkin R J. Jit breakup and mixing throat lengts for the liquid jit gas pump Journal of Fluids Engineering, Transactions of the ASME 96(3), pp 216226, (1974), DOI:10.1115/1.3447144.

$23 \mathrm{Hu}$ X Research of the design theory for liquid state jet pumps Scientia Sinica Series A:Mathematical, Physical, Astronomical \& Technical Sciences 26(2), pp. 214-224, (1983)

24 Sugati D Effendy M RANS study of the liquid jet gas ejector, In AIP Conference Proceedings American Institute of Physics Inc. Vol 1983 (1) p 020006, (2018), DOI: 10.1063/1.5046202. 